\title{
A Noble Method for Estimating Visual Greenery Ratio in Indoor Environment
}

\author{
Jung Nam Suh*, Kwang Jin Kim, and Yoonah Jang
}

Urban Agricultural Research Division, National Institute of Horticultural \& Herbal Science, Rural Development Administration, Wanju 55365, South Korea

\begin{abstract}
A noble method was developed for determining indoor greenery ratio through a digital camera and image analysis. Photographs with 10 different exposure compensations level were taken at indoor room using the function of "Partial color (green)" in a digital camera under varied indoor light conditions. RGB photographs were converted into Lab mode using [Image/Mode] function of Photoshop software program. The number of green pixels were calculated within 1 to 127 level (equivalent to -127 to -1 of Hunter a* value) through "Channel a" in [Histogram] function of the software. Greenery ratio was calculated by maximum green pixel number of an exposure compensation in relation to total pixel number, as maximum green pixels number would be obtained under a certain exposure compensation level when it minimizes achromatic color by partially excessive lower or higher light condition within certain area of photograph. When greenery ratio obtained from this method was compared with general greenery calculation of green area through manual selection, coefficient of determination was 0.9852 , indicating appropriate greenery ratio estimating method. When applied to vertical planter of veranda and office, greenery ratio varied at $0.53 \sim 14.96 \%$. In our experimental condition, error of the function, "Partial color" by the digital camera was within 0.013 , while the error of the function, "Channel a" by the software was within 0.005 .
\end{abstract}

Keywords: foliage plant, green interior, partial color, vertical planter

\section{Introduction}

The positive effects of introducing indoor plants to indoor living spaces have been found in many studies. The positive effects of indoor plants on indoor air quality and climate have been scientifically proven by many researchers since Wolverton et al. (1989), and decorating the interior with plants has been shown to improve the satisfaction about air quality by providing a subjective feeling about better air quality (Khan et al., 2005). Kaplan (1995) argues for the positive effects of indoor plants on the evolutionary side. In other words, the space in which the plant is located gives the feeling that people within it can be closer to the natural environment, making a positive impact on human physiology, thereby enhancing concentration recovery or productivity (Kaplan, 1995; Nieuwenhuis et al., 2014).

The introduction of indoor plants in the work space has been shown to improve the health, lessen discomfort feeling of office workers, reduce the number of days for sick leave (Fjeld, 2000), improve collaborative tasks and creative work 
(Shibata and Suzuki, 2002), and reduce tension and anxiety (Chang and Chen, 2005). On the other hand, when plants are introduced into the work space, workers feel that management pay attention to the welfare and satisfaction of their surroundings so that their satisfaction toward the managers and companies goes up, improving the concentration of work, job satisfaction, productivity and welfare consequently according to some studies (Parker, 1992; Bringslimark et al., 2007; Nieuwenhuis et al., 2014).

Because the indoor environment lacks sunlight, green foliage plants are mainly used rather than colorful flower plants (Briggs and Calvin, 1987). The visual greenery ratio is defined as the ratio of the area occupied by the green part of the plant in the human vision. The increase in the visual greenery ratio of the indoor space has a positive psychological effect (Choi et al., 2016), indicating that indoor landscape space offers positive image (Lee and Bang, 1996). Recently, several research and measurement methods have been reported to improve the visual greenery ratio in landscape architecture such as urban planning (Yang et al., 2009; Chen et al., 2015; Ding et al., 2016) There remains a problem in that the green area in the related photo data must be manually selected in the end. On the other hand, the study of visual greenery ratio in indoor space is insufficient, and the simple and reproducible measurement method has not been developed yet, which is a limiting factor in the study of quantitative processing of visual greenery ratio.

Conventional visual greenery ratio is determined by manually selecting the green area observed by the naked eye and calculating the number of pixels to obtain the ratio against the total pixels (Yang et al., 2009; Chen et al., 2015). However, there are problems that it consumes much time in measuring and a difference may occur between the workers. Meanwhile, Ding et al. (2016) reported an automatic visual greenery ratio measurement method, using the developed four-step algorithm, but there is a problem that under the $15.8 \%$ visual greenery ratio condition in outdoor environment, inaccuracy reached $21.4 \%$. Therefore, it is not suitable for use in indoor conditions with much lower visual greenery ratio. In this study, in order to develop a simple and reproducible method for measuring visual greenery ratio that can be applied to actual indoor space in uneven illuminance and light condition, conventional visual greenery ratio measurement and a comparative test were conducted on the office and vertical planter at the veranda to confirm the possibility of using the methods.

\section{Research Method}

The overall method of this study is to verify the validity of the method by analyzing the correlation between visual greenery ratios from the conventional visual greenery ratio method in which green area is selected manually after normal photographing and from the method of this study, green extraction photos. In addition, functional errors of the digital camera and computer programs were analyzed to check their availability.

\section{Photographing}

Under the maximum indoor lightening condition, the indoor panoramas of 17 offices and two vertical planters at the veranda were photographed diagonally from a $1.5 \mathrm{~m}$ tripod height at the entrance of the indoor space. Using a digital camera (Alpha a5000, 2015, Sony Korea, Thailand), the green area is selected manually to check the number of pixels by using the normal photo and color extraction (green) function and increasing exposure compensation (0-3) under automatic light metering conditions. Photographing was carried out at 10 different exposure compensation levels in total (Table 1). 
Table 1. Photographing condition in this experiment.

\begin{tabular}{lc}
\hline \multicolumn{1}{c}{ Camera mode } & Photographing condition \\
\hline Digital camera used & Sony alpha a5000 \\
Lens focal distance & $16 \mathrm{~mm}$ \\
ISO sensitivity & 100 \\
Shutter speed & Auto \\
Aperture & F10 \\
Number of pixel & $19,816,192$ \\
Compression & Fine \\
Exposure setting & Auto \\
White balance & Auto \\
Exposure compensation & 10 level $(0,0.3,0.7,1.0,1.3,1.7,2.0,2.3,2.7,3.0)$ \\
Effect mode & Picture effect (Partial color, green) \\
\hline
\end{tabular}

\section{Image processing and Pixel analysis}

The green extraction photographs taken under the 10 levels of exposure compensation were converted in image mode from RGB to Lab in Adobe Photoshop (ver.7.0). The 1 to 127 level (equivalent to -127 to -1 of Hunter a* value) through Channel a (green-red) in Histogram from the Lab photo was analyzed.

In order to measure the visual greenery ratio conventionally, the green part that can be visually confirmed by the Lasso and Magic wand functions of the above software was selected from the normal photo and the number of pixels on the histogram was obtained. After then, the ratio of the total pixels was calculated.

\section{Calculation of Visual greenery ratio}

The visual greenery ratio was calculated as the ratio of green pixels related to the total number of pixels. As for green extraction photo, visual greenery ration calculation used the number of pixels at the exposure compensation level with the maximum pixel numbers within 1 to 127 level through "Channel a" (green to red) taken under the condition with minimum number of pixels that become achromatic color (a value is 0 ) due to partially excessive lower of higher color condition under different light condition by location.

\section{Analysis on functional error}

To observe the error in the green extraction function of this digital camera, the standard error of the visual greenery ratio between the photographs as repeated processing areas was calculated after photographing five times at three different locations under the same condition.

In order to check the error of the calculation of the pixel number of green area of the software in the green extraction photograph, the number of pixels within 1 to 127 level through "Channel a" of the same photograph was repeated five times and analyzed to calculate the standard error of the visual greenery ratio. 


\section{Results and discussions}

When comparing visual greenery ratio calculated by this method with conventional method of measuring the ratio calculated by manual selection of green area, the result was $\mathrm{R}^{2}=0.9852$ (Fig. 1). Ding et al. (2016) reported an automatic visual greenery ratio measurement method that was $85.6 \%$ and $93.3 \%$ of the measured value respectively when the visual greenery ratio was $15.8 \%$ and $37.7 \%$ by conventional method of manual selection. This method allows measuring an average of $109.0 \%$ of visual greenery ratio against conventional method, with the exception of offices with less than $1 \%$ visual greenery ratio. Therefore, this is considered to be a more accurate measurement method for indoor environment which has a high possibility of error occurrence due to lower visual greenery ratio than outdoor.

The visual greenery ratio of the 17 offices surveyed according to this method varied from 0.53 to $14.96 \%$. In particular, the offices with vertical planter walls or partitions have a visual greenery ratio of $14.69 \%$ and $13.69 \%$, indicating that

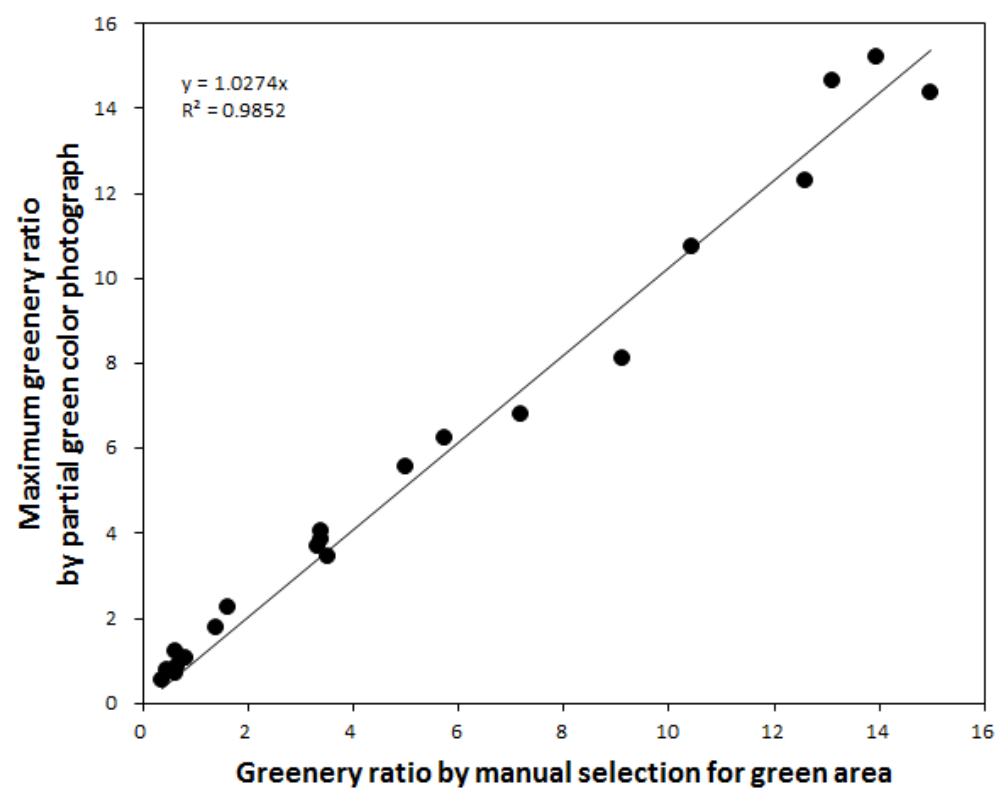

Figure 1. Relationship between greenery ratio calculated by manual selection for green area in normal photograph and from maximum greenery ratio calculated from partial green color photograph.

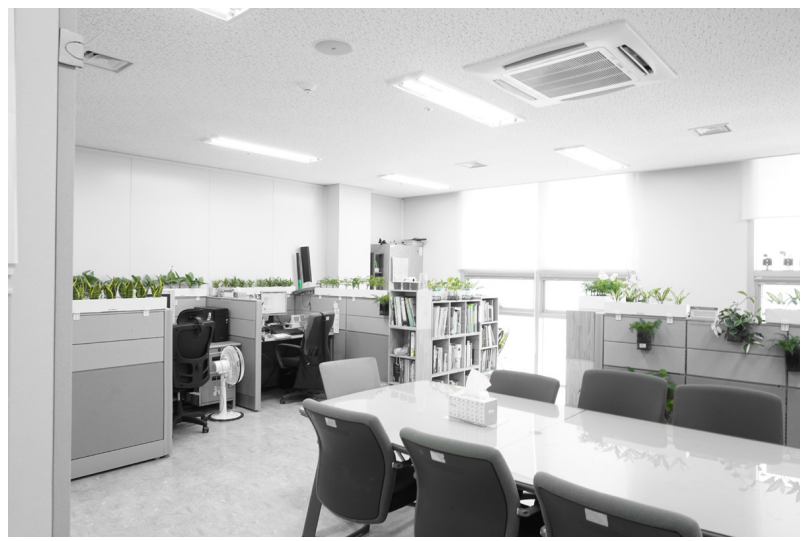

Greenery ratio - 3.86\%

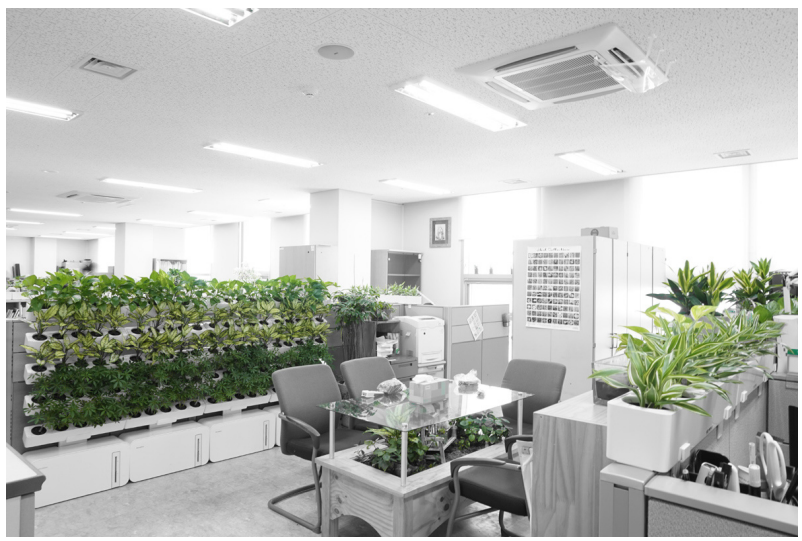

Greenery ratio $-14.69 \%$

Figure 2. Partial color (green) photographs showing the offices with or without vertical planters. 
vertical planter is useful in enhancing visual greenery ratio (Fig. 2). In addition, visual greenery ratio was high, $10.77 \%$ and $12.30 \%$, respectively, when the vertical planter in the veranda was introduced, which confirmed that installation of a vertical plant is effective way to increase the visual greenery ratio in various indoor environments.

Under the experimental conditions, the error of the function of "Partial color (green)" in a digital camera was 0.013 on average and the error of "Channel a" determination was 0.005 in Photoshop (Table 2). The reason why both errors are high in the office with low visual greenery ratio is presumed that the error occurred due to the function of "partial color (green)" in a digital camera and relatively higher number of boundary pixels compared to the total number of pixels during image processing by the software. It is consistent with the view of Ding et al. (2016), which suggests that the error increases due to the color mixing of the leaf boundary.

There are several considerations in measuring visual greenery ratio with this method. First, it is necessary to take a picture on a cloudy day in order to reduce the error caused by the severe difference in light intensity within the shooting area due to the natural light entering from the window. Second, reddish variegated parts can not be included in visual greenery ratio, subject to image processing in advance, while white or yellowish variegated parts of plants can be measured by being included in visual greenery ratio. Third, a book or drawer with greenery color other than the plant should be removed in advance or excluded during image process.

Despite these limitations, this method is useful for research on the visual greenery ratio of indoor living space as a simple and reproducible visual greenery ratio measurement method until a more sophisticated measurement method with less error is developed. In addition, it takes less work time than the image processing time in the previous study (Chen et al., 2015; Yang et al., 2009) and reproducibility is also stable since both camera error and software error are all low.

Although there are references to the types and amounts of introduced plants in the materials and methods of existing studies on the positive effects of indoor plant introduction (Adachi et al., 2000; Raanaas et al., 2011), there is no description about the numerical value of the accurate amount of visual greenery color. Therefore, it is almost impossible to conduct comparison study or reproduction experiment. In the future experiment to investigate the psychological change according to the introduction of the indoor plants, it will be guarantee detailed experimental description, if expressed the visual greenery ratio according to this method.

Aoki (1987) reported that when the visual greenery ratio changes in outdoor landscape was more than $25 \%$, it was perceived and when it was more than $50 \%$, most of the people were satisfied. However, there is no study on numerical changes of visual greenery ratio of indoor environment. In the future, it is expected that this visual greenery ratio measurement method will be used for the quantification of the process for the objective experimental environment

Table 2. Standard error of greenery ratio arisen from the function of "Partial color (green)" in a digital camera and calculated by "Channel a" determination in [Histogram] function of the Photoshop software.

\begin{tabular}{lcc}
\hline \multirow{2}{*}{$\begin{array}{c}\text { Greenery ratio in selected office } \\
(\%)\end{array}$} & $\begin{array}{c}\text { The function of "Partial color (green)" } \\
\text { in a digital camera }\end{array}$ & \begin{tabular}{c} 
Standard error \\
\cline { 2 - 3 }
\end{tabular} \\
\hline 3.26 & 0.020 & 0.009 \\
7.90 & 0.006 & 0.003 \\
14.96 & 0.012 & 0.002 \\
\hline Average & 0.013 & 0.005 \\
\hline
\end{tabular}

${ }^{\mathrm{z}}$ Standard error of 5 repeated photographs taken from the same condition.

${ }^{\mathrm{y}}$ Standard error of 5 repeated determination in the same photograph. 
description, the development of the planter for the increase of the visual greenery color in the indoor office space, and the study for the selection of suitable plants.

In conclusion, the visual greenery ratio in indoor environment was obtained by setting 10 different exposure compensation levels, using the green extraction camera function under the general indoor light conditions and then measuring the ratio of the maximum number of pixels within 1 to 127 level of "Channel a".

\section{Conclusion}

A new method for measuring visual greenery ratio of indoor space through digital camera and image analysis was developed and correlation with existing methods was analyzed. Using the camera function of extracting color (green), the photographs were taken diagonally at the indoor entrance, in 10 levels of increasing the exposure compensation $(0 \sim 3$ level) under the automatic light metering condition. The RGB photos taken were converted in image mode to Lab by using Photoshop software. The number of green pixels were calculated within 1 to 127 level (equivalent to -127 to -1 of Hunter $a^{*}$ value) through "Channel a" (green-red) in [Histogram] function of the Photoshop software. Greenery ratio was calculated by maximum green pixel number of an exposure compensation in relation to total pixel number, as maximum green pixel number would be obtained under a certain exposure compensation level when it minimizes achromatic color by partially excessive lower or higher light condition within certain area of photograph. The visual greenery ratio obtained by this method was $\mathrm{R}^{2}=0.9852$ as a result of the relationship between the visual greenery ratio obtained by manually selecting the green part and the measured value. Visual greenery ratio was $0.53 \sim 14.96 \%$ per measured indoor veranda and office. In this experiment, error of the function, "partial color" by the digital camera was within 0.013 while the error of the function, "Channel a" by Photoshop was within 0.005 .

\section{References}

Adachi, M., C.L.E. Rohde, and A.D. Kendle. 2000. Effects of floral and foliage displays on human emotions. HortTechnology 10(1):59-63.

Aoki, Y. 1987. Relationship between perceived greenery and width of visual fields, J. Jpn. Inst. Landsc. Archit. 51(1):1-10. Briggs, G.B. and C.L. Calvin. 1987. Indoor plants. New York, USA: John Wiley \& Sons, Inc.

Bringslimark, T., T. Hartig, and G.G. Patil. 2007. Psychological benefits of indoor plants in work places: Putting experimental results into context. HortScience 42(3):581-587.

Chang, C.Y. and P.K. Chen. 2005. Human response to window views and indoor plants in the workplace. HortScience 40(5):1354-1359.

Chen, Z., B. Xu, and B. Gao. 2015. Assessing visual green effects of individual urban trees using airborne Lidar data. Sci. Total Environ. 536:232-244. DOI: 10.1016/j.scitotenv.2015.06.142

Choi, J.Y., S.A. Park, S.J. Jung, J.Y. Lee, K.C. Son, Y.J. An, and S.W. Lee. 2016. Physiological and psychological responses of humans to the index of greenness of an interior space. Complementary Therapies in Medicine 28:37-43. DOI: 10.1016/j.ctim.2016.08.002

Ding, Y., T. Fukuda, N. Tabuki, T. Michikawa, and A. Motamedi. 2016. Automatic measurement system of visible greenery ratio using augmented reality. Proceedings of the 21st International Conference on Computer-Aided Architectural Design Research in Asia, Melbourne, Australia. Abstract retrieved from https://www.researchgate.net/publication/ 303952603_AUTOMATIC_MEASUREMENT_SYSTEM_OF_VISIBLE_GREENERY_RATIO_USING_AUGMEN TED_REALITY

Fjeld, T. 2000. The effect of interior planting on health and discomfort among workers and school children. HortTechnology 
10(1):46-52.

Kaplan, S. 1995. The restorative benefits of nature: Toward an integrative framework. J. Environ. Psychol. 15(3):169-182. DOI: 10.1016/0272-4944(95)90001-2

Khan, A.R., A. Younis, A. Riaz, and M.M. Abbas. 2005. Effect of interior plantscaping on indoor academic environment. J. Agric. Res. 43:235-242.

Lee, N.H. and K.J. Bang. 1996. The influence of the ratio of greenery on the visual preference in interior landscape. J. Korean Inst. Landsc. Archit. 24(2):13-24.

Nieuwenhuis, M., C. Knight, T. Postmes, and S.A. Haslam. 2014. The relative benefits of green versus lean office space: three field experiments. J. Exp. Psychol. Appl. 20(3):199-214. DOI: 10.1037/xap0000024

Parker, D.C. 1992. The corporate garden. In The Role of Horticulture in Human Well-Being and Social Development: A National Symposium. Timber Press, Portland, Oregon.

Raanaas, R.K., K.H. Evensen, D. Rich, G. Sjostrom, and G. Patil. 2011. Benefits of indoor plants on attention capacity in an office setting. J. Environ. Psychol. 31(1):99-105. DOI: 10.1016/j.jenvp.2010.11.005

Shibata, S. and N. Suzuki. 2002. Effects of the foliage plant on task performance and mood. J. Environ. Psychology 22:265-272. DOI: 10.1006/jevp.2002.0232

Wolverton, B.C., A. Johnson, and K. Bounds. 1989. Interior Landscape Plants for Indoor Air Pollution Abatement: Final Report-September 1989. National Aeronautics and Space Administration, John C. Stennis Space Centre.

Yang, J., L. Zhao, J. Mcbride, and P. Gong. 2009. Can you see green? Assessing the visibility of urban forests in cities. Landscape and Urban Planning 91(2):97-104. DOI: 10.1016/j.landurbplan.2008.12.004 